Classification

Physics Abstracts

$24.10-25.70$

\title{
Macroscopic dynamics of the fusion process
}

\author{
E. Tomasi, C. Grégoire, C. Ngô \\ DPh-N/MF, CEN Saclay, 91191 Gif sur Yvette Cedex, France
}

and B. Remaud

Institut de Physique, Université de Nantes, 2, rue de la Houssinière, 44072 Nantes, France

(Reçu le 4 décembre 1981, accepté le 22 décembre 1981)

\begin{abstract}
Résumé. - Nous avons calculé les sections efficaces de fusion pour différents systèmes à l'aide d'un modèle dynamique macroscopique. Ce modèle prend en compte la réorganisation des densités des deux noyaux ainsi que le transfert de nucléons. Les différents mécanismes suivant la capture du système dans la poche du potentiel d'interaction sont discutés. Les sections efficaces de fusion calculées sont en accord raisonnable avec les valeurs expérimentales.
\end{abstract}

\begin{abstract}
A macroscopic dynamical model has been used to calculate fusion cross sections for a wide number of systems ranging from ${ }^{16} \mathrm{O}+{ }^{27} \mathrm{Al}$ to ${ }^{40} \mathrm{Ar}+{ }^{165} \mathrm{Ho}$. This model takes into account the reorganization of the densities and the nucleon exchanges. The possible reaction mechanisms after capture of the system into the pocket of the interaction potential are discussed. Two processes contribute to fusion : compound nucleus formation and fast fission phenomenon. The calculated fusion cross sections are in overall agreement with the data.
\end{abstract}

1. Introduction. - The understanding of the fusion process is one of the oldest problems in heavy ion physics and it still keeps nowadays a high level of interest while it has been traditionally closely associated to compound nucleus formation, it seems now that the fusion cross section cannot always be identified to the compound nucleus cross section. Recent experiments might indicate the possible existence of a delayed deep inelastic component with a lifetime larger than the relaxation time associated to mass transfer [1]. As a matter of fact a symmetric mass distribution is observed similar to the one of the fission products following compound nucleus formation. Theoretical investigations have also shown this possibility [2-7] and the corresponding mechanism has been called fast fission [3-4]. It can be understood to occur as follows :

If the system is trapped in the entrance potential because of friction forces, it does not necessarily evolve to a compound nucleus configuration. Indeed, during the reorganization of the densities, the pocket in the potential energy surface where the system has been trapped, might disappear. This occurs for instance for large angular momenta when the fission barrier vanishes. Consequently the system reseparates again without forming a compound nucleus. However the mass distribution of these products will be symmetric if the time during which the system remains trapped is of the order or larger than the relaxation time for mass transfer. This kind of process has been extensively discussed in references [4-6]. 
In this letter we would like to use the model of references [4-6] to calculate the fusion cross sections for some systems where the experimental data are available. The reason to do this is the following :

We should aim at a model which explains simultaneously deep inelastic reactions and fusion. This means that we have to use a dynamical model. Indeed there are good static models to describe the experimental data for fusion but they are unable to describe the properties of deep inelastic reactions [8].

The dynamical models [9-11] which have been used up to now for a systematic investigation of the fusion data do not describe mass and isospin transfer. Furthermore they do not give a quantitative description of the energy loss observed in deep inelastic reactions and do not treat the fluctuations around the mean values of the macroscopic variables. As a matter of fact they only reproduce a limited number of properties for deep inelastic reactions. Furthermore a neglect of mass and isospin transfer as well as of the role of deformations might change the physical conditions under which the fusion process occurs.

2. Outline of the macroscopic model. - The model which we use has been described at length in references [4-6]. We shall just recall here a few important things which will be useful for our purpose.

The heavy ion collision is described by means of 4 macroscopic variables : two of them for the relative motion of the two ions and the two others for mass and isospin transfer. The deformations which are induced during the process are simulated by allowing a transition between a sudden potential [12], $V_{\mathrm{s}}$, in the entrance channel and an adiabatic potential [13], $V_{\mathrm{a}}$, in the exit channel. In this way the total interaction potential, $V$, is taken of the form :

$$
V=\chi(t) V_{\mathrm{s}}+(1-\chi(t)) V_{\mathrm{a}}
$$

with the boundary condition

$$
\chi(0)=1
$$

$\chi(t)$ is a reorganization parameter which can be rewritten from(1) as :

$$
\chi(t)=\frac{V-V_{\mathrm{a}}}{V_{\mathrm{s}}-V_{\mathrm{a}}}
$$

$t$ is of course the time. $\chi(t)$ is assumed to fulfil an equation of motion which is chosen a priori (Eq. (3) of Ref. [4]). If we would consider the parameter $\chi$ as an extra collective variable, this equation could be understood as leading to an overdamped motion (in the sense of Kramers [14]) for $\chi$.

In this model the dynamical evolution of the system is given by a density distribution in phase space of the collective degrees [15]. This distribution satisfies a transport equation the solution of which is a gaussian. The first moments of this distribution, which are the mean values of the collective variables, satisfy Newton equations with friction forces and follow classical trajectories. The second moments give the dispersion around these mean trajectories. The transport coefficients, which are the inputs necessary to solve the transport equation are the same as those of references $[5,6]$ except for the tangential friction coefficient $\gamma_{\theta}$. This latter quantity has been chosen here to be equal to $\gamma_{\mathrm{r}} / 2$ where $\gamma_{\mathrm{r}}$ is the radial friction coefficient. Indeed the relation $\gamma_{\mathrm{r}}=2 \gamma_{\theta}$ can directly be obtained from the window formula of reference [16]. With this relationship, the results of reference [6] relative to deep inelastic and fast fission properties remain almost unchanged. 
3. Fusion cross sections. - The dynamical evolution of the system is followed for different values of the impact parameter (or initial values of the orbital angular momentum $l$ ). In the interaction region the system loses kinetic energy due to friction forces. This energy loss can be large enough for the system to be trapped into the pocket of the interaction potential We call
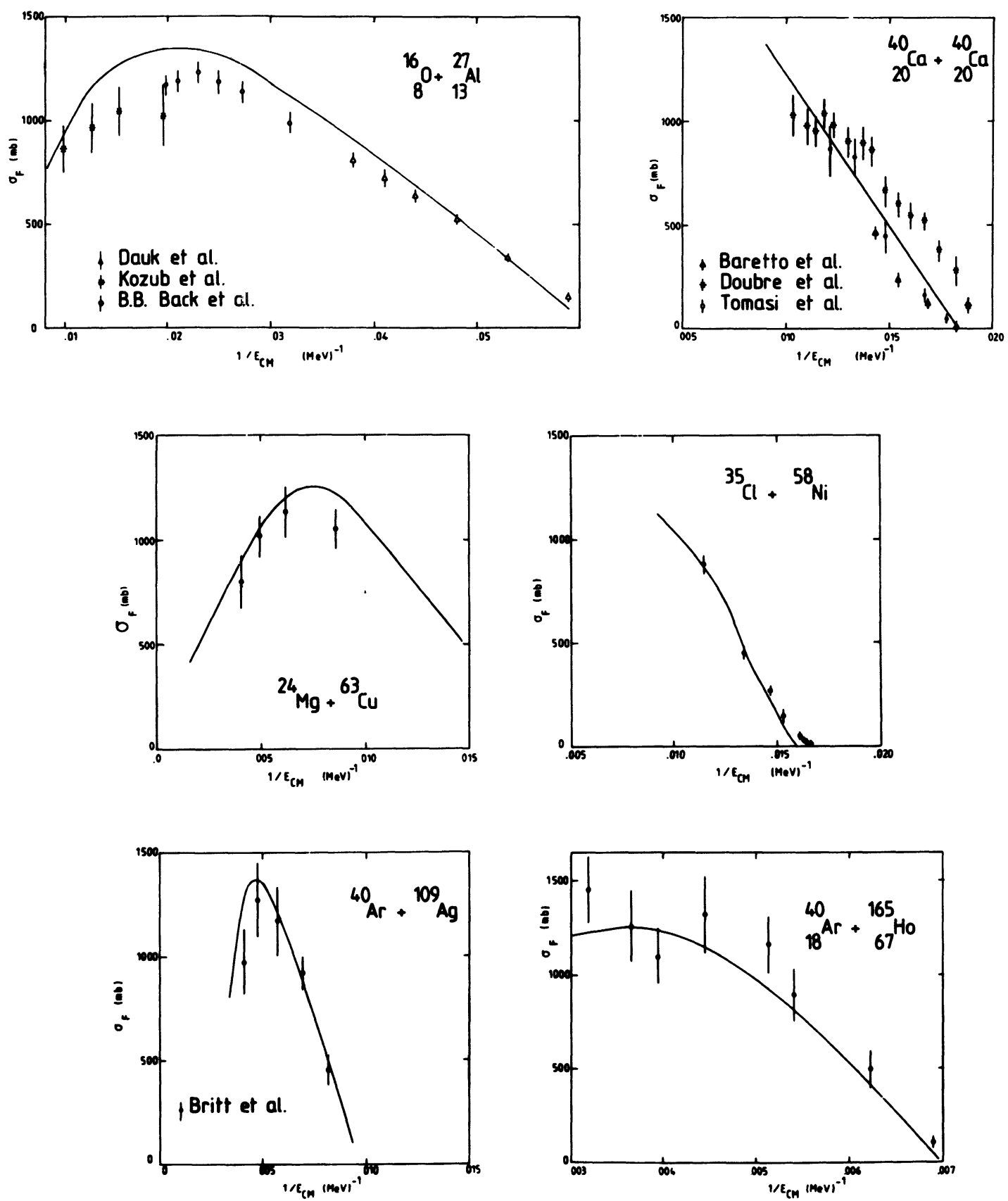

Fig. 1. - Excitation functions for the ${ }^{16} \mathrm{O}+{ }^{27} \mathrm{Al},{ }^{40} \mathrm{Ca}+{ }^{40} \mathrm{Ca},{ }^{24} \mathrm{Mg}+{ }^{63} \mathrm{Cu}, \quad{ }^{35} \mathrm{Cl}+{ }^{58} \mathrm{Ni}$, ${ }^{40} \mathrm{Ar}+{ }^{109} \mathrm{Ag},{ }^{40} \mathrm{Ar}+{ }^{165} \mathrm{Ho}$. The full curve is the result of our calculation. The experimental values are extracted from [1, 17-21]. 
fusion a trajectory which is trapped in the pocket of the entrance potential. We will see later on that such a trajectory can lead either to compound nucleus formation or to fast fission depending on the conditions. Trajectories which are not trapped but which correspond to a large energy loss are associated to deep inelastic reactions.

In figure 1 are shown the excitation functions for several systems for which a lot of experimental points are available : ${ }^{16} \mathrm{O}+{ }^{27} \mathrm{Al}\left(\right.$ Ref. [17]), ${ }^{40} \mathrm{Ca}+{ }^{40} \mathrm{Ca}\left(\operatorname{Ref}\right.$. [18]), ${ }^{35} \mathrm{Cl}+{ }^{58} \mathrm{Ni}(\operatorname{Ref}$ [19]), ${ }^{24} \mathrm{Mg}+{ }^{63} \mathrm{Cu}$ (Ref. [20]), ${ }^{40} \mathrm{Ar}+{ }^{109} \mathrm{Ag}$ (Ref. [21]) and ${ }^{40} \mathrm{Ar}+{ }^{165} \mathrm{Ho}$ (Ref. [1]). The full curves are the results of our calculation and the measured fusion cross sections are indicated together with their error bars. We note an overall agreement between the experimental data and our calculations. It should be noted that the calculations have been performed with the same set of parameters for the transport coefficients. When the fusion cross section $\sigma_{F}$ is plotted as a function of $1 / E_{\mathrm{CM}}$, the inverse of the centre-of-mass energy, there are three regimes at low, medium and high bombarding energies [8]. The data shown here show one or two of these regimes among the three which are possible.

At low bombarding energies the fusion process is mainly determined by the fusion barrier as it is also the case for static models. This is so because the energy loss almost compensates the decrease of the potential energy due to the loss of angular momentum [22]. The low energy tail, which is apparent for the $\mathrm{Cl}+\mathrm{Ni}$ system, cannot be reproduced by our model because we do not treat barrier transparency.

As the bombarding energy increases the energy loss due to friction forces is larger than the decrease of the potential energy due to the loss of angular momentum. As a matter of fact the fusion cross section is smaller than the one predicted by a static model using the fusion barrier. This is known in static approaches and was the reason to introduce the critical distance concept [23]. We see however here that there is no need to introduce the notion of critical distance to explain the data and that the decrease of $\sigma_{\mathrm{F}}$ at medium bombarding energies appears naturally as a result of friction forces. From our model the need of a critical distance has to be understood as an overpush necessary to have fusion due to the loss of kinetic energy before reaching the top of the fusion barrier.

At high bombarding energies we have a third regime in the plot $\sigma_{\mathrm{F}}$ versus $1 / E_{\mathrm{CM}}$ where the critical angular momentum saturates. This is illustrated for instance by the $\mathrm{Mg}+\mathrm{Cu}$ system. This can be understood in our model as follows : let $l_{1}$ be the angular momentum for which the pocket of the sudden interaction potential disappears. If there is no more a pocket the system cannot be trapped and fusion is not anymore possible. This means that the critical angular momentum remains bounded to a value $l_{\mathrm{CR}}^{\max }$. This value is larger than $l_{1}$ because tangential friction decreases the orbital angular momentum during the reaction. $l_{\mathrm{CR}}^{\max }$ can for instance correspond to the value $l_{1}$ before sticking.

4. What happens to the fusion trajectories. - For completeness we shall now examine what happens to the trajectories which have been trapped in the pocket of the sudden potential (an extensive discussion can be found in references $[5,6])$. Three cases can happen :

1) The system has a fission barrier and the saddle configuration in the adiabatic potential is located outside the bottom of the pocket of the sudden potential. In this case the system evolves to a compound nucleus. This occurs for light systems like $\mathrm{Ca}+\mathrm{Ca}, \mathrm{Mg}+\mathrm{Al}$ or $\mathrm{O}+\mathrm{Al}$.

2) The saddle configuration is more compact than the configuration corresponding to the bottom of the pocket. Then a compound nucleus cannot be formed and the system reseparates in two almost symmetric fragments. Such a process corresponds to fast fission or as in reference [7] to quasi-fission.

3) The system has no more a fission barrier due to angular momentum. In this case a compound nucleus cannot be formed and we observe fast fission. This process has been discussed at length 
in references [3-6]. This is the case for the $\mathrm{Ar}+\mathrm{Ho}$ and $\mathrm{Ar}+\mathrm{Ag}$ systems at large angular momentum.

5. Conclusion. - As a conclusion let us stress a little bit the features which are important, within our model, for the fusion process.

It turns out that the fusion process is determined in the early stages of the reaction. This means that the relevant potential energy surface to be considered is the sudden potential. This explains the success of the models based on it. As an important consequence a pocket is necessary to trap the system. This pocket disappears when the product $Z_{1} Z_{2}$ of the atomic numbers of the two ions is greater than 2500-3000. Consequently fusion cannot be observed for larger values of $Z_{1} Z_{2}$ [24]. The adiabatic potential has an importance only to determine the subsequent evolution of the fused system : either to a compound nucleus, when the system remains trapped, or to fast fission, when it can escape.

Finally it is interesting to consider the influence of charge equilibration. We know from deep inelastic reactions that this mode relaxes very quickly to equilibrium [25] but it needs first to be excited. A TDHF calculation [26] has shown that this occurs after the system has overcome the fusion barrier, i.e. according to our model after the stage where fusion has been decided. Our model would support this calculation as we can see in figure 2 for the $\mathrm{Cl}+\mathrm{Ni}$ system. Here we have performed two calculations : the full line corresponds to the case where charge equilibration is gradually excited after a point where the nuclear potential is deeper than $-3 \mathrm{MeV}$ whereas for the dashed line, charge equilibration gets gradually excited as soon as the densities overlap, i.e. before reaching the top of the fusion barrier.

We see that this has a great influence on the final results. Indeed, the excitation of the collective mode results in an increase of its kinetic energy that is not compensated by an equivalent potential energy loss. Due to the coupling with the other degrees of freedom, some energy is directly pumped

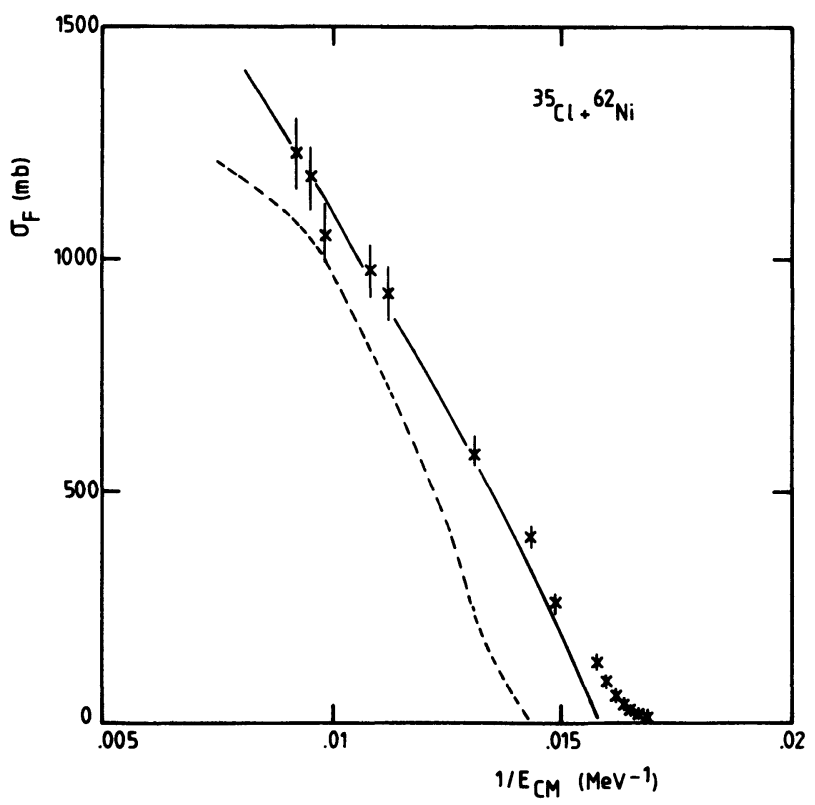

Fig. 2. - Calculated excitation function (full line) for the ${ }^{35} \mathrm{Cl}+{ }^{62} \mathrm{Ni}$ compared with the data of reference [19]. The dashed line is the result of a calculation with an early excitation of charge equilibration (see text). 
out from the relative kinetic energy. The more underdamped the collective mode, the stronger the extra damping induced in the relative motion. That is why the charge equilibration mode affects the fusion cross sections. Therefore, although it has a very short relaxation time, charge equilibrium seems to be excited after fusion is decided, i.e. after passing the top of the fusion barrier.

\section{References}

[1] Lebrun, C., Hanappe, F., Lecolley, J. F., Lefebvres, F., Ngô, C., Peter, J. and Tamain, B., Nucl. Phys. A 321 (1979) 207.

Borderie, B., Peter, J., Tamain, B., Agarwal, S., Girard, J., Grégoire, C., Matuszek, J., Ngô, C., Z. Phys. A 299 (1981) 263.

[2] Nörenberg, W., Riedel, C., Z. Phys. A 290 (1979) 335.

[3] Grégoire, C., Lucas, R., NGô, C., Schürmann, B. and Ngô, H., Nucl. Phys. A 361 (1981) 443.

[4] Grégoire, C., Ngô, C. and Remaud, B., Phys. Lett. 99B (1981) 17.

[5] Ngô, C., Grégoire, C. and Remaud, B., 3rd Adriatic europhysics study conference on the dynamics of heavy ion collisions, Hvar (Yugoslavia) 1981.

[6] Grégoire, C., Ngô, C. and Remaud, B., Preprint (1981). Submitted to Nucl. Phys. A.

[7] SwiateCKI, W. J., Nobel Symp. on high spin states, Orenas (Sweden) 1980.

[8] For a review see for instance NGô, C., Lectures given at the international summer school, Poiana Brasov (Roumania) 1980, proceedings p. 395.

[9] Gross D. and Kalinowski, H., Phys. Rep. 45 (1978) 175.

[10] Birkelund, R., Tubbs, L. E., Huizenga, J. R., De, J. N. and Sperber, D., Phys. Rep. 56 (1979) no 3.

[11] Gross, D., Nayak, R. C. and SatPathy, L., Z. Phys. A 299 (1981) 63.

[12] Ngô, C. Tamain, B., Galin, J., Beiner, M: and Lombard, R. J., Nucl. Phys. A 240 (1975) 353.

[13] Ledergerber, T. and Paul, H. C., Nucl. Phys. A 207 (1973) 1.

[14] Kramers, H., Physica VII (1940) 284.

[15] Hofmann, H. and Siemens, P. J., Nucl. Phys. A 275 (1977) 467.

[16] Blocki, J., Randrup, J., Swiatecki, W. J. and Tsang, C. F., Ann. Phys. 105 (1977) 427.

[17] Back, B. B., Betts, R. R., GaArde, C., Larsen, J. S., Michelsen, E. and Tai Kuang Hsi, Nucl. Phys. A 285 (1977) 317.

Dank, J., Lieb, K. P. and Kleinfeld, A. M., Nucl. Phys. A 241 (1975) 120.

Kozub, R. L., Lu, H. H., Miller, J. M. and Logan, D., Phys. Rev. C 11 (1975) 1497.

[18] Tomasi, E., Ardouin, D., Barreto, J., Bernard, V., Cauvin, B., Magnago, C., Mazur, C., Ngô, C., Piasecki, E. and Ribrag, M., to be published in Nucl. Phys.

Doubre, H., Gamp, A., Jacmart, J. C., Poffe, N., Roynette, J. and Wilczynski, J., Phys. Lett. 73B $\cdot(1978) 135$.

Barreto, J., Auger, G., Doubre, H., Langevin, M. and Plagnol, E., Ann. Rep., IPN (1980) Orsay and to be published.

BARRETO, J., thesis, Orsay (1980).

[19] Scobel, W., Gutbrod, H. H., Blann, M. and Mignerey, M. A., Phys. Rev. C 14 (1976) 1808.

[20] Borderie, B., Bimbot, R., Cabot, C., Gardes, D., Nowicki, L., Tamain, B., Grégoire, C., Ngô, C., Berlanger, M. and HanapPe, F., Z. Phys. A 298 (1980) 235.

[21] Britt, H. C., Erkkila, B. H., Stokes, R. G., Gutbrod, H. G., Plasil, F., Ferguson, R. J. and BlanN, M., Phys. Rev. C 13 (1976) 1483.

[22] Mosel, U., 3rd Adriatic europhysics study conference on the dynamics of heavy ion collisions, Hvar (Yugoslavia) 1981.

[23] Galin, J., Guerreau, D., Lefort, M. and Tarrago, X., Phys. Rev. C 9 (1974) 1018.

[24] NGô, H. and NGô, C., Nucl. Phys. A 348 (1980) 140.

[25] Gatty, B., Guerreau, D., Lefort, M., Tarrago, X., Galin, J., Cauvin, B., Girard, J. and NifeNECKER, H., Z. Phys. A 273 (1975) 65 and Nucl. Phys. A 253 (1975) 511.

[26] Bonche, P. and NGô, C., Phys. Lett. B 105 (1981) 17. 